\title{
Evolution of irradiated circumbinary disks
}

\author{
R. Günther, C. Schäfer, and W. Kley \\ Institut für Astronomie \& Astrophysik, Abt. Computational Physics, Auf der Morgenstelle 10, 72076 Tübingen, Germany \\ e-mail: rguenth@tat.physik.uni-tuebingen.de \\ Received 9 February 2004 / Accepted 23 April 2004

\begin{abstract}
We study the evolution and emission of circumbinary disks around close classical T Tauri binary systems. High resolution numerical hydrodynamical simulations are employed to model a system consisting of a central eccentric binary star within an irradiated accretion disk. A detailed energy balance including viscous heating, radiative cooling and irradiation from the central star is applied to calculate accurately the emitted spectral energy distribution.

Numerical simulations using two different methods, the previously developed Dual-Grid technique with a finite difference discretization, and the Smoothed Particle Hydrodynamics method are employed to compare the hydrodynamical features and strengthen our conclusions.

Physical parameters of the setup are chosen to model the close systems of DQ Tau and AK Sco. Using the self-consistent models, we are able to fit the observed spectral energy distributions by constraining parameters such as disk mass, density profile and radial extension for those systems. We find that the incorporation of irradiation effects is necessary to obtain correct disk temperatures.
\end{abstract}

Key words. accretion, accretion disks - stars: binaries: spectroscopic - hydrodynamics - methods: numerical

\section{Introduction}

From observations it is known that a large fraction of young stars are in binaries. The formation of binary systems by close interactions in a star forming region was studied numerically by Bate et al. (2002). Typically, a circumbinary accretion disk surrounding the double star is formed during this process. Here we concentrate on a later phase and model the hydrodynamic evolution of the circumbinary disk around close binary stars having a separation of only a fraction of an AU. This is inspired by observations of the spectroscopic binaries DQ Tau and AK Sco for which there is also a spectral energy distribution (SED) available. The latter system AK Sco was reanalyzed recently by Alencar et al. (2003).

In a previous paper (Günther \& Kley 2002) we modeled the evolution of circumbinary disks and compared fully developed circumbinary disks and their properties with observational data. That work extended previous computations by Artymowicz \& Lubow (1996) and Rozyczka \& Laughlin (1997) by solving explicitly a time dependent energy equation including the effects of viscous heating and radiative cooling. We investigated both the structure and dynamics of the disk as well as the gas flow in the close vicinity of the binary star. To that purpose we utilized a newly developed method which enables us to cover the whole spatial domain called the Dual-Grid method. For the first time we performed long-time integration of the complete system covering several hundred orbital periods of the binary and compared the properties of the evolved systems with observational data such as spectral energy distributions in the infrared and optical bands and accretion rates estimated from observed luminosities.

In this paper we extend the model by accounting for irradiation effects which are important for passive accretion disks such as the disks of DQ Tau and AK Sco, according to observational data. Additionally, refined observational data is available from Alencar et al. (2003) for the AK Sco system which allows to better constrain the parameters of the system by our numerical simulations.

In the next section we present the equations that are being solved and the refined radiative balance model. The overall layout of the physical model is the same as in Günther \& Kley (2002). Then we proceed with some remarks on the generation of the spectra from the simulation data (Sect. 3). After that in Sect. 4 we describe the two-dimensional simulation setup and proceed to the new results in Sect. 5. We summarize and conclude in Sect. 6.

\section{Equations}

The evolution of the disk is given by the two-dimensional $(r, \varphi)$ evolutionary Navier-Stokes equations for the density $\Sigma$, the velocity field $\boldsymbol{u} \equiv\left(u_{r}, u_{\varphi}\right)$, and the temperature $T$. In a coordinatefree representation the equations read

$\frac{\partial \Sigma}{\partial t}+\nabla \cdot(\Sigma \boldsymbol{u})=0$ 
$\frac{\partial \Sigma \boldsymbol{u}}{\partial t}+\nabla \cdot(\Sigma \boldsymbol{u} \cdot \boldsymbol{u})=-\nabla P-\Sigma \nabla \Phi+\nabla \cdot \boldsymbol{T}$

$\frac{\partial \Sigma \varepsilon}{\partial t}+\nabla \cdot[(\Sigma \varepsilon+P) \boldsymbol{u}]=-\Sigma \boldsymbol{u} \cdot \Phi-\nabla \cdot(\boldsymbol{F}-\boldsymbol{u} \cdot \boldsymbol{T})$.

Here $\varepsilon=c_{\mathrm{v}} T+1 / 2 u^{2}$ is the specific total energy, $P$ is the vertically integrated (two-dimensional) pressure $P=R \Sigma T / \mu$ with the midplane temperature $T$ and the mean molecular weight $\mu$, which can be obtained by solving the Saha rate equations for hydrogen dissociation and ionization and helium ionization. $\boldsymbol{F}$ and $\boldsymbol{T}$ are the radiative flux and viscous stress tensor, respectively.

The gravitational potential $\Phi$ generated by the binary stars is given by

$\Phi=-\sum_{i=1,2} \begin{cases}\frac{G M_{i}}{\left|\boldsymbol{r}-\boldsymbol{r}_{i}\right|} & \text { for }\left|\boldsymbol{r}-\boldsymbol{r}_{i}\right|>R_{*} \\ \frac{G M_{i}\left(3 R_{*}^{2}-\left|\boldsymbol{r}-\boldsymbol{r}_{i}\right|^{2}\right)}{2 R_{*}^{3}} & \text { for }\left|\boldsymbol{r}-\boldsymbol{r}_{i}\right| \leq R_{*}\end{cases}$

where $\boldsymbol{r}_{i}$ are the radius vectors to the two stars, and $R_{*}$ is the stellar radius assumed to be identical for both stars. The second case in Eq. (4) gives the potential inside a star with radius $R_{\star}$ having a homogeneous (constant) density.

\subsection{Viscosity and disk height}

The effects of viscosity are contained in the viscous stress tensor $\boldsymbol{T}$. Here we assume that the accretion disk may be described as a viscous medium driven by some internal turbulence which we approximate with a Reynolds ansatz for the stress tensor. The components of $\boldsymbol{T}$ in different coordinate systems are spelled out explicitly for example in Tassoul (1978). A useful form for disk calculations considering angular momentum conservation is given in Kley (1999).

For the kinematic shear viscosity $v$ we use an $\alpha$-model (Shakura \& Sunyaev 1973) of the form

$v=\alpha c_{\mathrm{s}} H$,

where $c_{\mathrm{s}}$ and $H$ are the local sound speed and vertical height, respectively. The local disk height $H(\boldsymbol{r})$ is computed from the vertical hydrostatic equilibrium which yields

$H(\boldsymbol{r})=\left(\sum_{i=1,2} \frac{G M_{i}}{c_{\mathrm{s}}^{2}\left|\boldsymbol{r}-\boldsymbol{r}_{i}\right|^{3}}\right)^{-\frac{1}{2}}=\left(\sum_{i=1,2} H_{i}^{-2}(\boldsymbol{r})\right)^{-\frac{1}{2}}$.

This can be split into the single star disk heights given by $H_{i}(\boldsymbol{r})=c_{\mathrm{s}} \sqrt{\frac{\left|\boldsymbol{r}-\boldsymbol{r}_{i}\right|^{3}}{G M_{i}}}$. The sound speed is given here by $c_{\mathrm{s}}=$ $R T / \mu$.

\subsection{Radiative balance}

The influence of radiative and viscous effects on the disk temperature is treated in a local vertical balance. The balance equation for the internal and radiative energy considering these effects reads

$\frac{\partial\left(\Sigma c_{\mathrm{V}} T\right)}{\partial t}=Q_{\mathrm{diss}}-Q_{\mathrm{rad}}+Q_{\mathrm{stars}}$ where $Q_{\text {diss }}$ and $Q_{\text {rad }}$ denote the viscous dissipation and radiative losses. $Q_{\text {stars }}$ is the irradiation heating by the stars, which is considered for the optically thick regions of the circumbinary disk. This inclusion of irradiation effects is done in a simple geometric fashion due to the lack of explicit treatment of the vertical structure of the disk in these two-dimensional calculations. For the viscous dissipation we use the vertically averaged expression

$Q_{\mathrm{diss}}=\boldsymbol{u} \cdot \nabla \boldsymbol{T}=\frac{1}{2 \Sigma v} \operatorname{Tr}\left(\boldsymbol{T}^{2}\right)$

where $\boldsymbol{T}$ is the viscous stress tensor. For the radiative transport we use

$Q_{\mathrm{rad}}=-\nabla \cdot \boldsymbol{F}_{0}-\frac{\partial F_{z}}{\partial z}$

where $\boldsymbol{F}_{0}$ is the flux vector in the $z=0$ plane. Here we consider only the losses in the vertical direction, i.e. $\boldsymbol{F}_{0}=0$, a standard approximation in accretion disk theory. Integration over the vertical direction yields

$Q_{\mathrm{rad}}=2 F_{\mathrm{rad}}=2 \sigma_{\mathrm{B}} T_{\mathrm{eff}}^{4}$

with the local effective (surface) temperature $T_{\text {eff }}$. Following Hubeny (1990) this temperature is related to the midplane temperature $T$ by the LTE gray solution for the vertical structure of accretion disks. Simplifying this relation we get

$T^{4}=\frac{3}{4} T_{\mathrm{eff}}^{4}\left[\frac{1}{2} \tau+\frac{1}{\sqrt{3}}+\frac{1}{3 \tau}\right]$

The optical depth is calculated via (Ruden \& Pollack 1991)

$\tau=\frac{1}{2} \kappa \Sigma$

using an interpolated opacity $\kappa(\rho, T)$ adapted from Lin \& Papaloizou (1985).

\subsubsection{Irradiation}

For the heating from the stars we use

$Q_{\text {stars }}=A \sigma_{\mathrm{B}} T_{*}^{4} \sum_{i}\left(\frac{R_{*}}{r_{i}}\right)^{2}$,

where $T_{*}$ is the effective blackbody temperature of the stars, $R_{*}$ is the stellar radius and $r_{i}$ are the distances of the stars to the local patch. The factor $A$ honors the effective surface the flux operates on. This is obtained by reconstructing a surface from the radially varying pressure scale height. Self-shadowing is taken into account by setting $A$ to zero for back-faces.

Numerical tests have shown that with the inclusion of irradiation effects we observe oscillatory behavior of the temperature near to the edge of the gap due to self-shadowing effects as also found by Dullemond (2000). But we can overcome this specific instability by using appropriate time substepping for the radiative balance. This suggests that if the radiative balance is imposed using a time relaxation process the self-shadowing instability does not occur. This may be either because the cooling timescale is not within the region of linear instability or because of non-linear effects exposed by the relaxation mechanisms. 


\section{Spectra generation}

Spectral energy distributions from the disk emission can be computed by integrating the flux of the blackbody radiation from the disk surface. This is done decoupled from the numerical simulation as a postprocessing step. We follow Adams et al. (1988), and compute the flux at the observer via

$F_{v}=\frac{\cos i}{D^{2}} \int_{r_{0}}^{R_{d}} \int_{0}^{2 \pi} B_{v}[T(r, \varphi)]\left(1-\mathrm{e}^{-\tau(r, \varphi)}\right) r \mathrm{~d} \varphi \mathrm{d} r$

where $i$ is the inclination of the disk, $D$ the distance to the observer and $\tau$ the line-of-sight optical depth through the disk, which can be approximated using the opacity $\kappa, \tau(r, \varphi)=\frac{\kappa \Sigma(r, \varphi)}{\cos i}$. The surface disk temperature $T$ is estimated using the optical depth of the disk as in the radiative balance process.

This integration is limited to regions outside of the stellar cores as temperatures and densities inside the cores are not modeled correctly. The stellar emission is accounted for by adding two blackbody spectra for appropriate effective stellar temperatures. To compare with observational data all of the generated spectra are reddened according to an extinction $A_{v}$ suggested by the cited references using the method from Cardelli et al. (1989).

\section{Simulation setup}

We start with an axisymmetric circumbinary disk around the binary system with an initial gap of a width that is determined by simulations including the gap formation process. This results in gap widths about three to four times the binary separation radius consistent with results from Artymowicz \& Lubow (1994). This initial configuration is then evolved for several orbital periods, so the circumstellar environment can form and the whole system settles into a quasi-periodic state.

Initial density and temperature distributions for the disk are taken from power-law fits of Mathieu et al. (1997) and Alencar et al. (2003). This initial surface temperature is relaxed to a midplane temperature satisfying Eq. (11).

The stellar temperatures and radii specify the flux observed, and that transfered into the disk. These parameters cannot be fitted independently but need to be fixed for the irradiation of the disk. This is why we use stellar radii as suggested by Mathieu et al. (1997) and Alencar et al. (2003) and fit the effective temperatures $T_{*}$ according to the observed spectral energy distributions by assuming simple blackbody spectra. Direct matching of the stellar spectra is a good approximation as can be seen in Fig. 1, where we display the AK Sco disk with the binary stars projected using the estimated inclination of $63^{\circ}$. This demonstrates that occultation of the stars by the circumbinary disk is not to be expected.

The overall azimuthally averaged circumbinary disk shape does not change significantly on timescales of the orbital period of the binary system. Induced by the gravitational torques of the central binary a nice a spiral structure develops in the inner regions of the disk as can be seen in Fig. 2.

Also one can observe periodic formation of spiral arms from the edge of the circumbinary disk going down to the circumstellar environment (Fig. 3) connected to the binary orbital

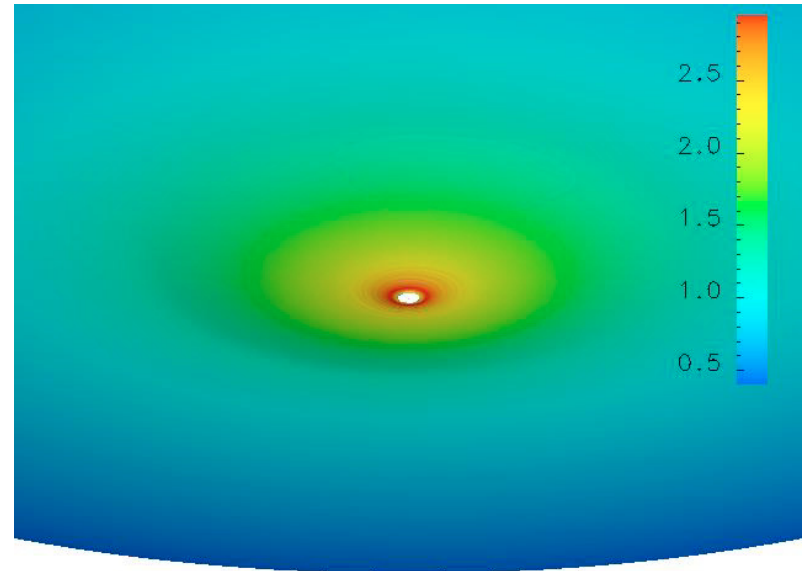

Fig. 1. Visible setup of the AK Sco disk system using the estimated inclination of $63^{\circ}$. The disk shape is modeled according to pressure scale height, gray scale coding is logarithmic surface density in $\mathrm{g} / \mathrm{cm}^{2}$. The disk extent is $12 \mathrm{AU}$.

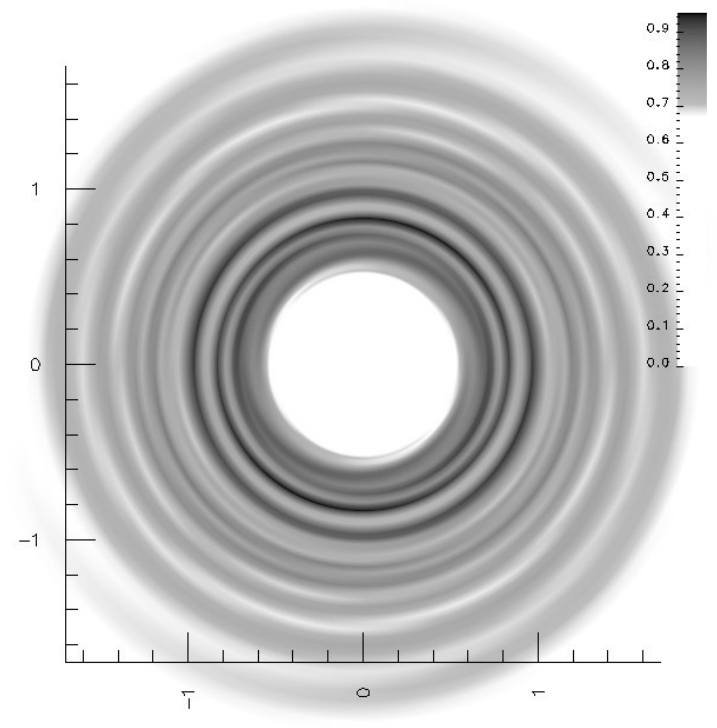

Fig. 2. Linear surface density $\left(\mathrm{g} / \mathrm{cm}^{2}\right)$ of the inner circumbinary disk of the DQ Tau system after 50 orbital periods. Scaling is in AU. The spiral structure in the circumbinary disk induced by the binary is clearly seen.

period. The quasi-periodic state of the circumstellar environment of the eccentric system is characterized by the following phases:

1. Going from periastron to apastron, material is accreted to the circumstellar environment from the circumbinary disk and the remaining material in the gap.

2. Around apastron phase circumstellar envelopes/disks have been formed.

3. Approaching periastron, these circumstellar disks are torn off due to gravitational torques exerted on the disks by the stars, and a part of the mass is accreted onto the stars.

This leads to periodic accretion events which is both confirmed from observations (Basri et al. 1997) showing periodic brightening events and from numerical simulations. A more detailed 


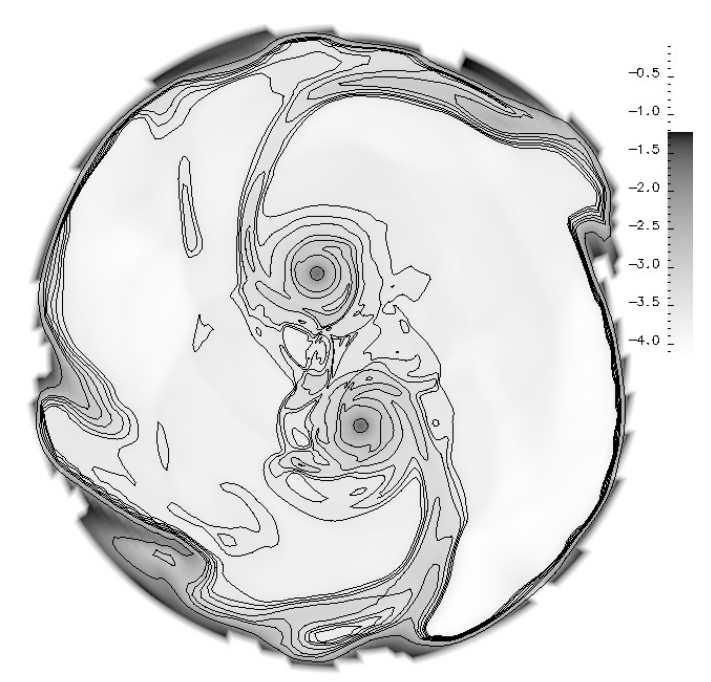

Fig. 3. The spiral arms onto the circumstellar environment for the DQ Tau system after 50 orbital periods. The gray scale follows logarithmic surface density annotated with iso-lines. Plotted is the region inside the circumbinary disk gap which is located at $0.4 \mathrm{AU}$. The binary is in apastron phase.

discussion on the disk dynamics and accretion can be found in Günther \& Kley (2002).

\subsection{Dual-Grid and SPH}

We additionally have performed Smoothed Particle Hydrodynamics (SPH) test calculations which cover only the inner part of the simulation domain to corroborate the results of the Dual-Grid technique. The initial setup consists of two circumstellar disks in Keplerian motion around the individual stars in apastron. Similar to the grid based calculations we evolve this system for a couple of orbital periods to reach a quasi-stationary state. For detailed descriptions of the SPH method see, e.g., Benz (1990) and Monaghan (1992). The SPH simulations include only radiative cooling and viscous heating and not the irradiation from the stars, as these calculations are primarily intended to support the results obtained by the Dual-Grid technique.

\section{Results}

Grid based calculations have been performed on two different regions of the whole system. First, high resolution $(110 \times 204)$ calculations extending only over the circumbinary disk (0.412 AU for AK Sco and 0.3-80 AU for DQ Tau) have been performed to evolve these regions in higher resolution and for a long time. Second, high resolution $(180 \times 247$ for the $r-\varphi$ grid, $101 \times 101$ for the Cartesian grid) calculations extending over the circumstellar disks and up to the very inner region of the circumbinary disk (up to 1.2 AU for AK Sco and up to 1.0 AU for DQ Tau) have been run to allow comparisons with the SPH calculations. Finally, low resolution $(128 \times 91,37 \times 37)$ simulations of the whole system using the Dual-Grid technique have been performed to show that indeed these regions decouple in the timescales we are interested in.

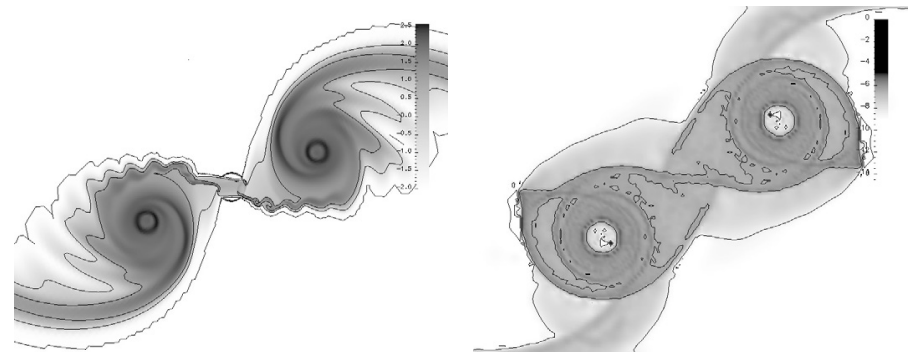

Fig. 4. Circumstellar disks logarithmic density structure for Dual-Grid simulation (left) and SPH simulation (right) while approaching periastron. The binary separation is about $0.1 \mathrm{AU}$.

\subsection{Dual-Grid and SPH}

The Dual-Grid technique was developed to overcome limitations of using cylindrical coordinates for accretion disks, where the whole domain including the origin needs to be covered (Günther \& Kley 2002). To demonstrate that the interpolation scheme used between the Cartesian and the cylindrical grid is accurate enough for modeling close binary circumstellar disks, we performed comparison runs using SPH.

Simulations are set up to initially contain two circumstellar disks around the individual stars with a mass of $10^{-8} M_{\odot}$ and a disk profile according to $\Sigma \propto r^{-3 / 2}$. This system is then evolved for a few orbital periods to truncate the disks to the right size and go into a quasi-stationary equilibrium. Dual-Grid calculations were performed on a $180 \times 247$ sized $r-\varphi$ grid and a $101 \times 101$ sized Cartesian grid, while the SPH calculations instrumented 150000 particles.

Figure 4 shows the structure of the disks for Dual-Grid and SPH calculations after the same evolution time. In both simulations one can see similar circumstellar material features, namely

- spiral structure inside the circumstellar material;

- spiral waves going off the circumstellar region;

- a bar-like connection between both circumstellar regions.

Thus we can conclude that with the Dual-Grid calculations we see the same features across the origin as with the SPH calculations. The Dual-Grid technique is applicable to simulation of circumbinary systems including the origin and the inner circumstellar regions.

\subsection{Irradiation effects}

For motivating the inclusion of irradiation effects, we first analyze the individual contribution of the different terms in the radiative balance (Eq. (7)), and the pressure work $p \nabla \cdot \boldsymbol{u}$, to the temperature change rate. The azimuthally averaged rate of the temperature change at quasi-stationary equilibrium of the DQ Tau system is plotted in Fig. 5 for the inner regions of the disk and Fig. 6 for the outer regions. Here, visc denotes the rate from the viscous heating, irr the rate from the irradiation process, cool the emission contribution and $\mathrm{pdV}$ the rate from the pressure work. Note, that the inner edge of the disk is approximately at $r=0.4$ AU for this model. 


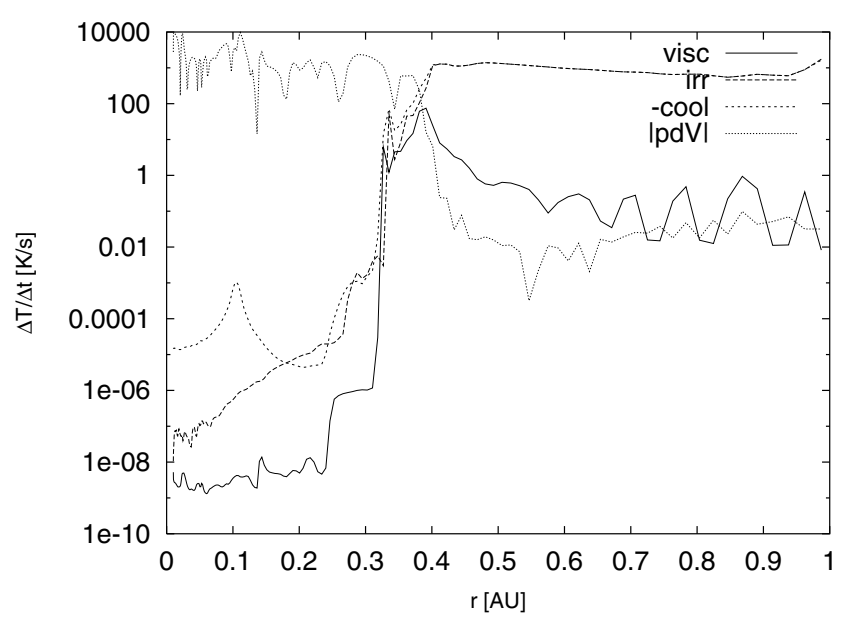

Fig. 5. Contribution of the different heating/cooling terms to the rate of temperature change (DQ Tau after 50 orbital periods). Only the inner part of the system is displayed. The irradiation irr and the radiative cooling -cool match exactly beyond 0.4 AU.

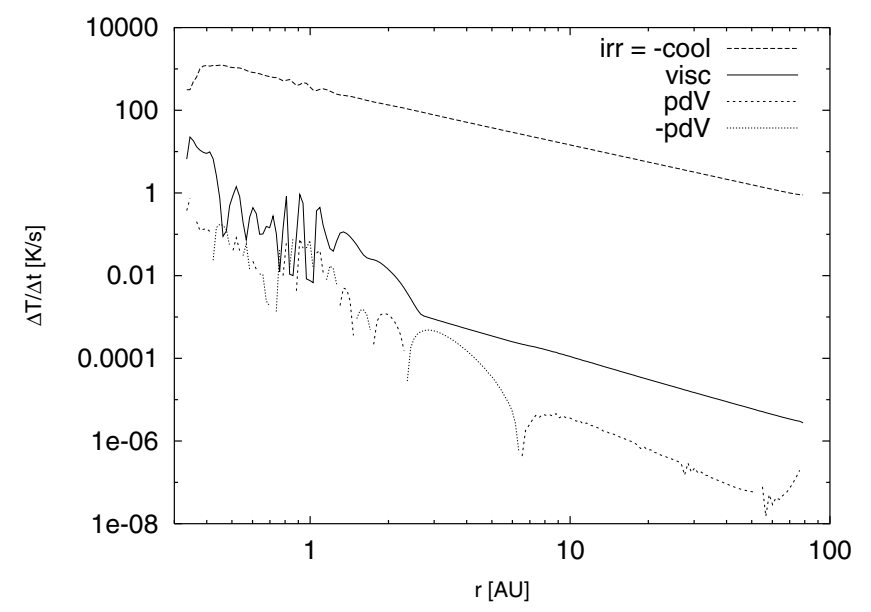

Fig. 6. Contribution of the different heating/cooling terms to the rate of temperature change (DQ Tau after 50 orbital periods). Plotted is the outer circumbinary disk part of the system. The irradiation by the central stars and the radiative cooling match exactly (topmost dashed line).

As one can easily infer from Fig. 6 the circumbinary disk is dominated by irradiation effects (which in fact match the cooling rates exactly), and as such qualifies as a passive disk. The effects due to viscous heating and pressure work can be neglected here.

In the inner optically thin part the pressure work is dominating due to the dynamic motion in this region caused by the gravitational torques exerted by the eccentric binary. One can also see that the emitted flux in this region is negligible compared to the flux emitted by the circumbinary disk.

\subsection{Circumbinary disks}

We model the two close spectroscopic binary systems with circumbinary disks, DQ Tau and AK Sco, which are both of $\mathrm{T}$ Tauri type. The physical parameters for the systems are taken from Alencar et al. (2003) for AK Sco and Mathieu et al. (1997)
Table 1. Parameters for DQ Tau (Mathieu et al. 1997).

\begin{tabular}{ll}
\hline \hline$P$ & $15^{\mathrm{d}} .8043 \pm 0.0024$ \\
$e$ & $0.556 \pm 0.018$ \\
$i$ & $23^{\circ}$ \\
$a \sin i$ & $0.024 \pm 0.009 \mathrm{AU}$ \\
$M_{\mathrm{A}} \sin ^{3} i$ & $0.033 \pm 0.002 M_{\odot}$ \\
$M_{\mathrm{B}} \sin ^{3} i$ & $0.033 \pm 0.002 M_{\odot}$ \\
$q$ & $1.0 \pm 0.03$ \\
$T_{*}$ & $4000 \mathrm{~K}$ \\
$R_{*}$ & $1.785 R_{\odot}$ \\
$d$ & $140 \mathrm{pc}$ \\
$M_{\mathrm{d}}$ & $0.002-0.02 M_{\odot}$ \\
$R_{\mathrm{d}}$ & $50 \mathrm{AU}$ \\
$A_{\mathrm{v}}$ & 2.0 \\
\hline
\end{tabular}

Table 2. Parameters for AK Sco (Alencar et al. 2003).

\begin{tabular}{ll}
\hline \hline$P$ & $13.609453 \pm 0.000026$ \\
$e$ & $0.4712 \pm 0.0020$ \\
$i$ & $63^{\circ}$ \\
$a \sin i$ & $0.14318 \pm 0.00005 \mathrm{AU}$ \\
$M_{\mathrm{A}} \sin ^{3} i$ & $1.064 \pm 0.007 M_{\odot}$ \\
$M_{\mathrm{B}} \sin ^{3} i$ & $1.050 \pm 0.007 M_{\odot}$ \\
$q=M_{\mathrm{B}} / M_{\mathrm{A}}$ & $0.987 \pm 0.005$ \\
$T_{*}$ & $6500 \pm 100 \mathrm{~K}$ \\
$R_{*}$ & $1.59 \pm 0.35 R_{\odot}$ \\
$d$ & $152 \mathrm{pc}$ \\
$M_{\mathrm{d}}$ & $0.005 M_{\odot}$ \\
$R_{\mathrm{d}}$ & $40 \mathrm{AU}$ \\
$A_{\mathrm{V}}$ & $0.5 \pm 0.1$ \\
\hline
\end{tabular}

for DQ Tau. The relevant information for the simulations has been summarized in Tables 1 and 2. Both stars are assumed to have the same effective temperature $T_{*}$ and the same radius $R_{*}$.

For both the DQ Tau and the AK Sco system we shall see missing flux at around $5 \times 10^{13}$, resp. $10^{14} \mathrm{~Hz}$ which can be accounted to the modeling of the T Tauri type stars emission as a simple blackbody.

\subsubsection{DQ Tau}

From full simulations including the gap formation process we infer an initial gap with a radius of 0.4 AU for the DQ Tau system. The stars combined effective blackbody temperature is fitted manually to $3500 \mathrm{~K}$ to match the observations. Starting from the parameters presented in Table 1 and power-law fits from Mathieu et al. (1997) we adjust the initial density distribution power-law index, the disk mass and its extension to best match the observed SED after going to quasi-stationary state.

The SED of DQ Tau can be fitted best with a thin disk extending to $80 \mathrm{AU}$ which has an unusually flat surface density distribution $\Sigma \propto r^{-0.25}$ and a total disk mass of $7 \times 10^{-4} M_{\odot}$. This is less mass and a larger disk radius as obtained by Mathieu et al. (1997). After fifty orbital periods, the equilibrium 


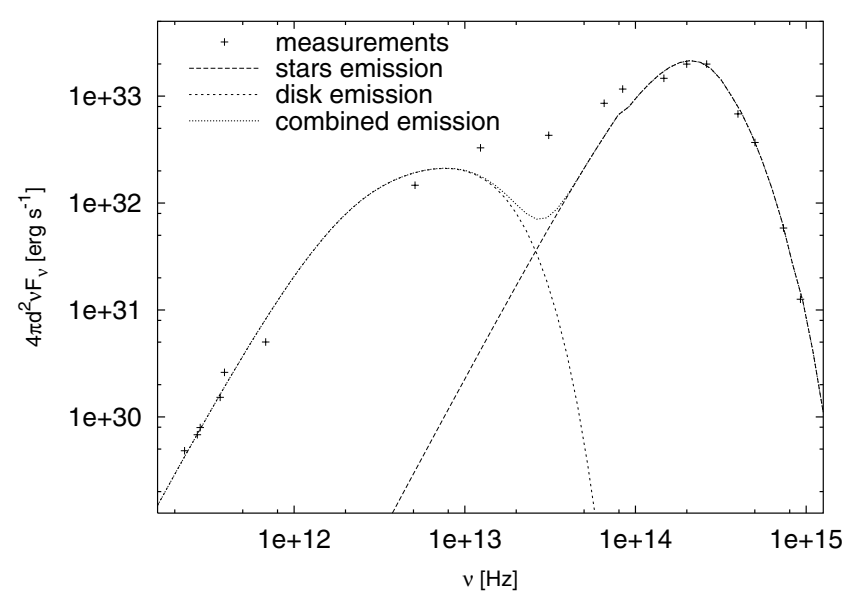

Fig. 7. Computed SED of DQ Tau. Crosses show observational data taken from Mathieu et al. (1997).

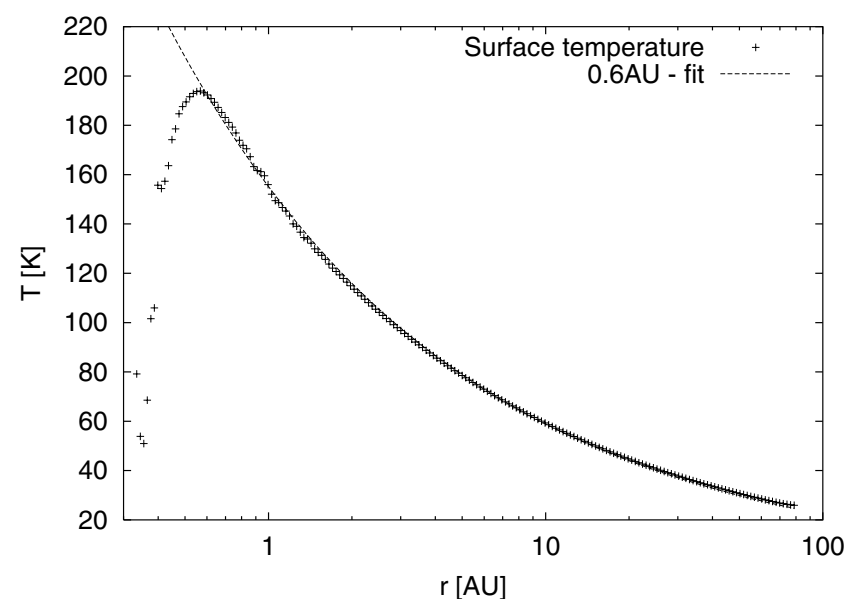

Fig. 8. Azimuthally averaged surface temperature of the equilibrium DQ Tau circumbinary disk.

surface temperature distribution of the circumbinary disk follows $T(r)=155 \mathrm{~K} r^{-0.42}$ as can be seen in Fig. 8 .

\subsubsection{AK Sco}

In Fig. 16 of Alencar et al. (2003) the authors propose a circumbinary disk mass of $M_{\mathrm{d}}=0.02 M_{\odot}$ and a disk extend of $R_{\mathrm{d}}=12 \mathrm{AU}$ for the best fit to the observed SED. The surface density distribution is that of a minimum mass solar nebula as in the used model (Chiang \& Goldreich 1997), so $\Sigma(r) \sim r^{-1.5}$. In Fig. 9 the emission of a model evolved with these parameters imposed initially is displayed.

Clearly visible is the excess flux of the model in the infrared (around $5 \times 10^{13} \mathrm{~Hz}$ ) which we can reduce by lowering the disk mass and slightly flattening the disk density profile. Using a disk mass of $M_{\mathrm{d}}=0.01 M_{\odot}$ and an initial density profile according to $\Sigma(r) \sim r^{-1.0}$ we obtain a spectrum as seen in Fig. 10.

For the AK Sco system the stars combined effective blackbody temperature is best fitted to the observations by assuming $T_{*}=6500 \mathrm{~K}$. In a quasi-stationary state after about fifty orbital periods of the binary the surface temperature can be fitted to

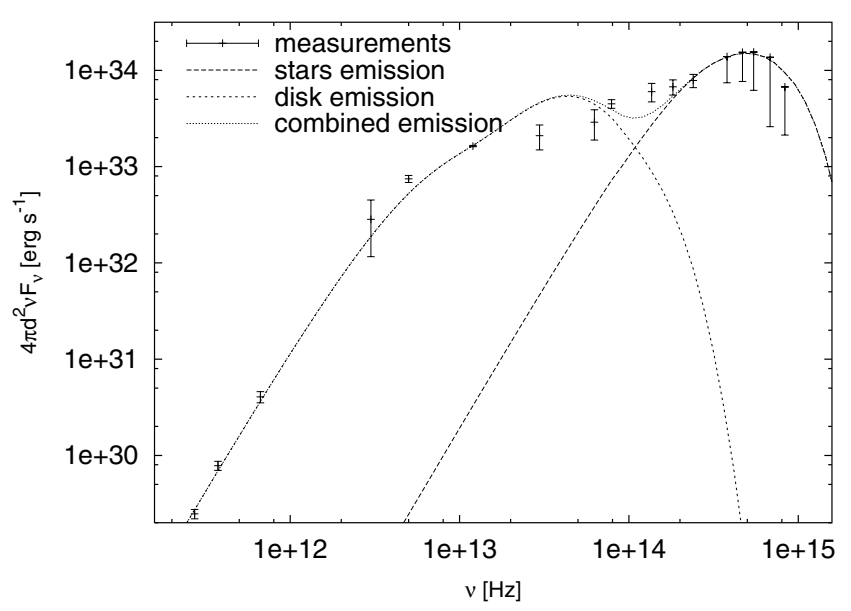

Fig. 9. Computed SED of AK Sco with parameters and observational data (crosses with error-bars) taken from Alencar et al. (2003).

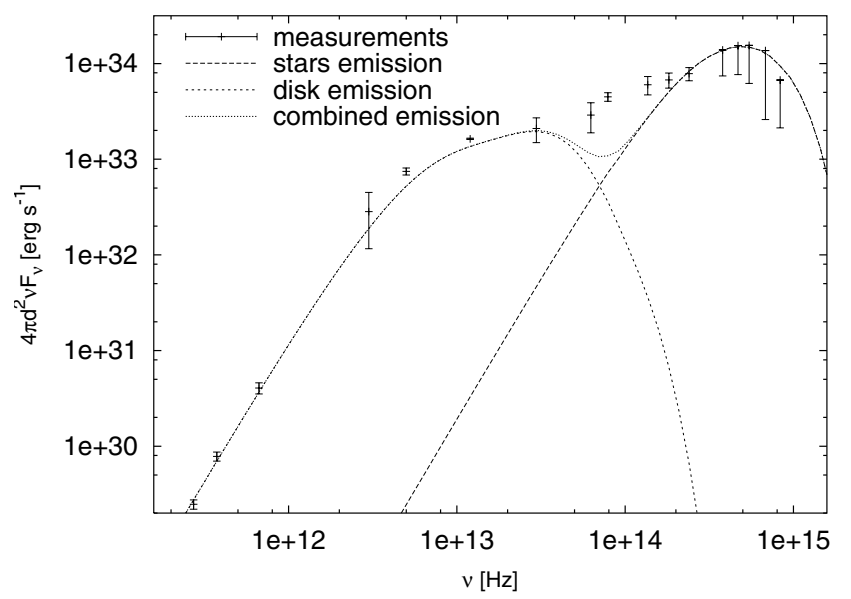

Fig. 10. Computed SED of the AK Sco system with best-fit parameters. Crosses with error-bars show observational data taken from Alencar et al. (2003).

the power-law $T(r)=264 \mathrm{~K} r^{-0.47}$ for $r>2$ AU and a surprisingly steep linear behavior in the region between one and two $\mathrm{AU}(T(r)=617 \mathrm{~K}-215 \mathrm{~K} r)$ as shown in Fig. 11. This is due to the different opacities in the dense region of the disk and compared to the case of DQ Tau three orders of magnitude higher maximum surface density which is related to the AK Sco disk being much smaller and more massive.

We note that our models are not able to fit the higher flux observed in the SED of both systems in the region between the disk and stellar contributions. This may be attributed to line emission features (Alencar et al. 2003) which cannot be modeled by our simulations.

\subsection{Circumstellar disks}

For the region inside the circumbinary disk gap we see a tiny amount of warm gas and streamers feeding circumstellar disk like structures from the inner circumbinary disk edge. Due to the low mass this material does not contribute to the continuum part of the observed spectral energy distributions but rather would show up in lines and the UV part of the spectra. 


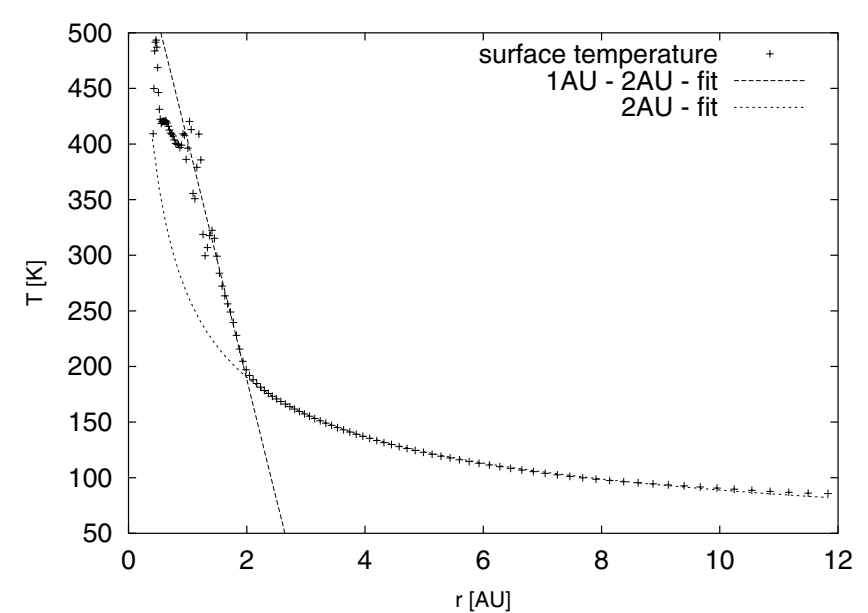

Fig. 11. Azimuthally averaged surface temperature of the equilibrium AK Sco circumbinary disk.

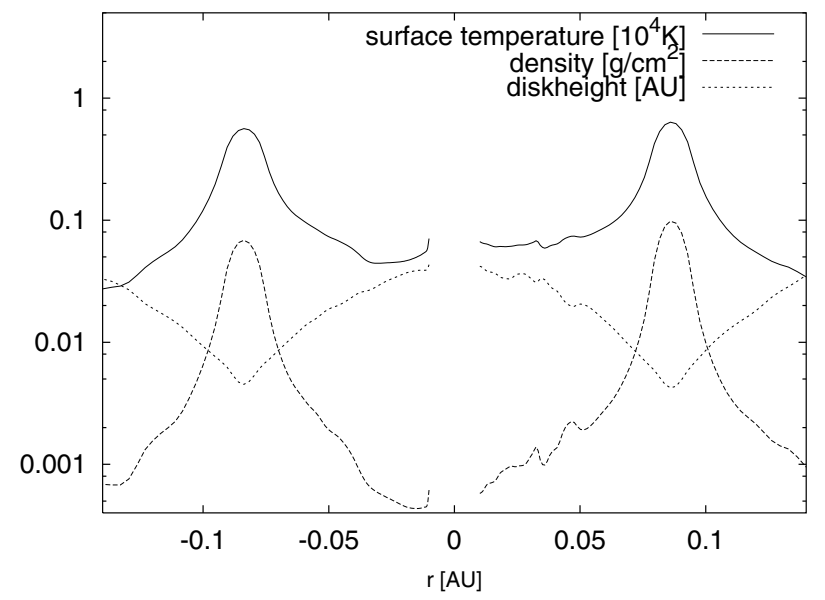

Fig. 12. Profiles of the circumstellar material along the connecting line of the binary at apastron in the system of DQ Tau (stellar radii $0.008 \mathrm{AU})$.

Also, the observed periodic brightening in the light-curve from the systems originate here, which is believed to originate from periodic accretion events. These periodic events of accretion have been confirmed by Artymowicz \& Lubow (1996), Rozyczka \& Laughlin (1997) and Günther \& Kley (2002) through numerical simulations.

The circumstellar disk like structures around DQ Tau form through accumulation of material in the stellar gravitational potential. Without removing any material through an accretion process, disk profiles according to Fig. 12 form. They cannot be identified with a classical accretion disk due to shape and size as one can see from Fig. 13 which shows the circumstellar material and velocity distribution. They rather would form sort of an envelope, but this remains to be investigated in threedimensional calculations.

\section{Conclusion}

We model the circumbinary disks of DQ Tau and AK Sco and their circumstellar environment including emitted spectra by applying two-dimensional hydrodynamical simulations

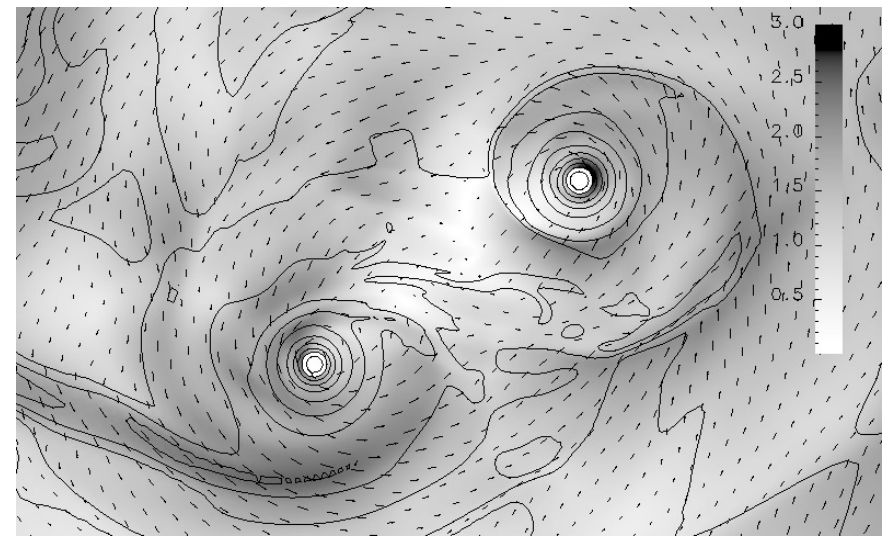

Fig. 13. Circumstellar material in the DQ Tau system after 41 orbital periods, corresponding to Fig. 12. Gray scale coding is velocity magnitude annotated with vector glyphs, isolines are equally spaced logarithmic surface density. The stellar cores are the white circles.

of high accuracy and resolution. The models include a selfconsistent approximative vertical energy transport including irradiation from the stars using detailed opacities and equation of state. The numerical resolution we have reached by now is so fine that the individual stars in close binaries are already resolved by typically about $10 \times 10$ grid-cells for high-resolution calculations.

We find that with suitable variations of

1. the initial surface density profile $\Sigma \sim r^{-d}$;

2. the circumbinary disk mass $M_{\mathrm{d}}$;

3. and the circumbinary disk extent $R_{\mathrm{d}}$,

the spectral energy distributions of DQ Tau and AK Sco can be fitted well to the observational data only if irradiation effects are included.

The circumbinary disk settles into a quasi-stationary state after around 50 orbital periods of the binary with a temperature distribution following approximately $T(r) \propto r^{-0.45}$ for larger radii in both systems. We find that irradiation plays the dominant role for the heating balance in these outer regions. In the inner part of the disk just beyond the inner gap, the additional heating due to the tidal effects is important, particularly for small disks around tight binaries, such as AK Sco.

For the present model parameter we do not find any indication for a previously observed self-shadowing instability of the disk due to the stellar irradiation (Dullemond 2000). A proper numerical treatment of the strongly non-linear cooling is required to prevent artificial numerical instabilities, which is achieved by using a time-relaxation scheme as opposed to directly solving for the equilibrium.

Future models of this kind need to include a more detailed treatment of the stellar boundary layers as well as a more detailed model of the accretion process. We believe that doing full radiative transfer in two dimensions will not improve our results, but going to three dimensions with full radiative transfer included will possibly enable us to obtain more accurate spectra from the circumbinary disk gap region with its thin material. We then may be able to compare in more detail with observed light curves and phase dependent spectra. 
Acknowledgements. This work was funded by the German Science Foundation (DFG) under SFB 382 Simulation physikalischer Prozesse auf Höchstleistungsrechnern.

\section{References}

Adams, F. C., Shu, F. H., \& Lada, C. J. 1988, ApJ, 326, 865 Alencar, S. H. P., Melo, C. H. F., Dullemond, C. P., et al. 2003, A\&A, 409, 1037

Artymowicz, P., \& Lubow, S. H. 1994, ApJ, 421, 651

Artymowicz, P., \& Lubow, S. H. 1996, ApJ, 467, L77

Basri, G., Johns-Krull, C. M., \& Mathieu, R. D. 1997, AJ, 114, 781

Bate, M. R., Bonnell, I. A., \& Bromm, V. 2002, MNRAS, 336, 705

Benz, W. 1990, in Numerical modelling of nonlinear stellar pulsations problems and prospects, 269
Cardelli, J. A., Clayton, G. C., \& Mathis, J. S. 1989, ApJ, 345, 245

Chiang, E. I., \& Goldreich, P. 1997, ApJ, 490, 368

Dullemond, C. P. 2000, A\&A, 361, L17

Günther, R., \& Kley, W. 2002, A\&A, 387, 550

Hubeny, I. 1990, ApJ, 351, 632

Kley, W. 1999, MNRAS, 303, 696

Lin, D. N. C., \& Papaloizou, J. 1985, in Protostars and Planets II, 981 Mathieu, R. D., Stassun, K., Basri, G., et al. 1997, AJ, 113, 1841

Monaghan, J. J. 1992, ARA\&A, 30, 543

Rozyczka, M., \& Laughlin, G. 1997, in Accretion phenomena and related outflows, ASP Conf. Ser., 121, IAU Coll., 163, 792

Ruden, S. P., \& Pollack, J. B. 1991, ApJ, 375, 740

Shakura, N. I., \& Sunyaev, R. A. 1973, A\&A, 24, 337

Tassoul, J. 1978, Theory of rotating stars, Princeton Series in Astrophysics (Princeton: University Press) 